\title{
Multiple-Ring Based Modeling and Simulation of Wideband Space-Time-Frequency MIMO Channels
}

\author{
Xiang Cheng ${ }^{\dagger}$, Cheng-Xiang Wang ${ }^{\dagger}$, and David I. Laurenson ${ }^{\dagger \dagger}$ \\ ${ }^{\dagger}$ Joint Research Institute for Signal and Image Processing, Heriot-Watt University, EH14 4AS, Edinburgh, UK. \\ ${ }^{\dagger \dagger}$ Joint Research Institute for Signal and Image Processing, University of Edinburgh, EH9 3JL, Edinburgh, UK. \\ Email: xc48@hw.ac.uk, cheng-xiang.wang@hw.ac.uk, dave.laurenson@ed.ac.uk
}

\begin{abstract}
In this paper, based on the concept of the Tapped Delay Line (TDL) structure, we first propose a new wideband multiple-ring Multiple Input Multiple Output (MIMO) channel reference model for macro-cell scenarios. It is shown that the proposed model can easily match any given or measured power delay profile (PDP) and has the ability to jointly consider the angle of arrival (AoA), angle of departure (AoD), and time of arrival (ToA). From the proposed model, we derive the closed-form expression of the 3-Dimensional (3-D) Space-Time-Frequency (STF) correlation function (CF) for each tap, considering the interaction of the temporal, spatial, and frequency correlations. Finally, based on the proposed reference model, a deterministic simulation model is then proposed.
\end{abstract}

\section{INTRODUCTION}

To successfully design a MIMO wireless system, it is necessary to have accurate and reliable MIMO channel models. Well-known one-ring MIMO channel models [1] and [2], with a ring of effective scatterers around the Mobile Station (MS), are widely used for the analysis and design of narrowband MIMO systems in macro-cell scenarios due to their close agreement with the measured data [3] and mathematical tractability. To reach the high demand for highspeed communications, wideband MIMO systems have been suggested in many communication standards. However, the one-ring structure that assumes effective scatterers located on a one-ring is overly simplistic and thus unrealistic for modeling wideband channels [4]. Therefore, in [4] the authors for the first time extended the narrowband one-ring model to a wideband model by extending the location of effective scatterers on a one-ring to a circular-ring. However, it is not trivial to use this model to match any given or measured PDP since many parameters need adjustments via a complicated approach. In contrast to [4], in [5] the one-ring model was extended to a wideband application by dividing the one-ring into several segments in terms of different ToAs. This makes the model easier to use to match specific PDPs. However, the one-ring structure was still applied in this model, which causes this model to exhibit an unrealistic structure in that certain ToAs (or propagation delays) are always related to a certain proportion of AoAs. Moreover, neither of the two models considered the interaction of the AoA, AoD, and ToA, the importance of which was described in [7] and investigated the STF correlations for each time-bin signal (i.e., each tap in a TDL model). Finally, the derived STF CFs based on those two models cannot reveal the frequency dependency on AoA statistics, which is a unique characteristic of wideband MIMO channels [8].

The goals of this paper are three-fold. First, we propose a new wideband MIMO channel model that represents a reasonable compromise between physical reality and analytical tractability. The proposed model uses a concentric multiplering instead of one-ring around the MS to avoid the one-ring structure. Also, to easily match any specified or measured PDP, the model utilizes a virtual confocal multiple-ellipse to construct a TDL structure. Moreover, this model has the ability to consider the interaction of the AoA, AoD, and ToA. Secondly, from the proposed model, a closed-form expression of the STF CF for each time-bin signal is derived. For simplicity, the spatial and frequency correlations were assumed to be independent in [4] and [5]. On the contrary, in this paper we derive the STF CF by taking into account the dependency between them. Therefore, the derived STF $\mathrm{CF}$ can explicitly relate the frequency correlation to various propagation parameters (e.g., mean AoA and angle spread). Moreover, based on the derived $\mathrm{CF}$, we reveal the inherent frequency correlation within the spatial correlation, which is important for the design of MIMO frequency diversity systems [9] and [10]. Since the proposed wideband model is a reference model that assumes an infinite number of effective scatterers, it cannot be implemented directly in practice. Therefore, the third goal of this paper is to derive an ergodic statistical (deterministic) simulation model based on the proposed reference model. Closed-form expressions are provided for the STF CF of the simulation model. The statistical properties of our simulation model are verified by comparing with the corresponding statistical properties of the reference model.

\section{A NeW Wideband Multiple-Ring Based MiMO CHANNEL REFERENCE MODEL}

A narrowband one-ring MIMO model is suitable for describing a narrowband channel in macro-cell scenarios, where the Base Station (BS) is elevated and unobstructed, while the MS is surrounded by a large number of local scatterers. For narrowband systems, since the propagation delays $\tau_{n}^{\prime}$ of all $N(N \rightarrow \infty)$ incoming waves are much smaller than the data symbol duration $T_{s}$, i.e., $\tau_{\max }^{\prime}=\max \left\{\tau_{n}^{\prime}\right\}_{n=1}^{N} \ll T_{s}$, the delay differences caused by different local scatterers randomly around the MS can be neglected in comparison to $T_{s}$. Therefore, it is reasonable to use the effective scatterers located 
on a one-ring instead of the real local scatterers to construct the low complexity one-ring model at the minor expense of accuracy [3]. However, in high data rate wideband systems, $T_{s}$ is much smaller than that in narrowband systems. In this case, the propagation delay differences cannot be neglected and thus the channel becomes a wideband channel. Therefore, the onering structure violates the basic characteristics of wideband channels as mentioned in [4].

To extend one-ring model to wideband applications in macro-cell scenarios, the primary task is to modify the oversimple one-ring structure. To this end, we replace the one ring of effective scatterers by concentric multiple rings of effective scatterers around the MS to capture the basic characteristics of wideband channels. To make our model easier to match any specified or measured PDP, we utilize the confocal multiple virtual ellipses with the BS and MS located at the foci to represent the TDL structure, where different delays correspond to different virtual confocal ellipses (i.e., taps). Note that the total number of virtual confocal ellipses, $L$, and the values of major axes $a$ of different ellipses are determined according to the specified or measured PDP. The newly developed structure is shown in Fig. 1. For clarity, Fig. 1 only presents the effective scatterers from three concentric rings (the total number of concentric rings in the $l$ th tap is $\Lambda_{l}$ ) belong to the $l$ th tap $(l=0,1, \ldots, L-l)$. Notice that the total number and radii of the concentric multiple rings for different taps (i.e., $\Lambda_{l}$ and $R_{l, i}$ ) can be different in terms of different propagation environments for different delays. Therefore, the new wideband model with the appropriate number and major axes of virtual confocal multiple ellipses (i.e., $L$ and $a$ ), and the appropriate number and radii of concentric multiple rings (i.e., $\Lambda_{l}$ and $R_{l, i}$ ) should be suitable for any macrocell scenario. We assume that uniform linear antenna arrays are used with $n_{B S}=n_{M S}=2$ antennas. The symbols $\delta_{T}$ and $\delta_{R}$ designate the antenna element spacing at the BS and MS, respectively, and $D_{T R}$ denotes the distance between the BS and MS. The effective scatterers are located on $\sum_{l=0}^{L-1} \Lambda_{l}$ rings with radii $R_{l, i}\left(i=0,1, \ldots, \Lambda_{l}-1\right)$. It is usually assumed that the assumption $D_{T R} \gg R_{l, i} \gg \max \left\{\delta_{T}, \delta_{R}\right\}$ is fulfilled. The multi-element antenna tilt angles are denoted by $\alpha$ and $\beta$. The MS moves with speed in the direction determined by the angle of motion $\gamma$. The angle spread seen at the BS is denoted by $\Theta_{l . i}$, which is related to $R_{l, i}$ and $D_{T R}$ by $\Theta_{l, i} \approx \arctan \left(R_{l, i} / D_{T R}\right) \approx R_{l, i} / D_{T R}$.

The received complex impulse response at the carrier frequency $f_{c}$ for the link $A_{q}-A_{o}$ can be expressed as:

$$
h_{o q}\left(t, \tau^{\prime}\right)=\sum_{l=0}^{L-1} h_{l, o q}(t) \times \delta\left(\tau^{\prime}-\tau_{l}^{\prime}\right)
$$

with $o=1,2, \ldots, n_{M S} l=0$ and $q=1,2, \ldots, n_{B S}$, where $h_{l, o q}(t)$ and $\tau_{l}^{\prime}$ denote the complex time-variant tap coefficient and the discrete propagation delay of the $l$ th tap, respectively. Similar to the concept of effective scatterers in the narrowband onering model, the concept of an effective cluster is introduced in the new wideband multiple-ring model. From Fig. 1, it is obvious that the position of the effective cluster $S_{l, i}$ is specified by the intersection of the virtual ellipses and multiple rings, and the $l$ th tap includes $2 \Lambda_{l}$ effective clusters. The mean angle of the effective cluster in each tap is $\varphi_{l, i, r}(r=1,2)$ and the corresponding angle spread is $\Delta \varphi_{l, i}$ as illustrated in Fig. 1. Therefore, the effective cluster can be completely determined by $\varphi_{l, i, r}$ and $\Delta \varphi_{l, i}$. Note that the angular range of $\varphi_{l, i, r}$ in each tap is over $[0,2 \pi)$, which means the effective cluster can be located around the MS over $[0,2 \pi)$ for each tap. The setting of these two parameters follows a fixed rule. To establish this rule, firstly, we need to define the propagation delay subintervals $G_{l}$. The propagation delay interval $G=\left[0, \tau_{\max }^{\prime}\right]$ is partitioned into $L$ mutually disjoint sub-intervals $G_{l}$. Here, we utilize the definition of subintervals as [6]

$$
G_{l}= \begin{cases}{\left[0, \Delta \tau_{l+1}^{\prime} / 2\right),} & l=0 \\ {\left[\tau_{l}^{\prime}-\Delta \tau_{l}^{\prime} / 2, \tau_{l}^{\prime}+\Delta \tau_{l+1}^{\prime} / 2\right),} & l=1,2, \ldots, L-2 \\ {\left[\tau_{l}^{\prime}-\Delta \tau_{l}^{\prime} / 2, \tau_{\max }^{\prime}\right],} & l=L-1\end{cases}
$$

where $\Delta \tau_{l}^{\prime}=\tau_{l}^{\prime}-\tau_{l-1}^{\prime}$ and $\tau_{\max }^{\prime}=2 R_{L-1, \Lambda_{L-1}-1} / c(c$ is the speed of light). The propagation delay $\tau_{l}^{\prime}$ of the $l$ th tap can be expressed according to the corresponding AoA $\phi_{l, i, r}^{\prime R}(r=1,2)$ as $\tau_{i}^{\prime} \approx \tau_{i, \max }^{\prime}\left(1+\cos \phi_{l, i, r}^{\prime R}\right) / 2$ [5]. Solving this equation for $\phi_{l, i, r}^{\prime R}$ gives $\phi_{l, i, r}^{\prime R}= \pm \arccos \left(2 \tau_{l}^{\prime} / \tau_{i, \max }^{\prime}-1\right)$. According to (2), the expression of $\phi_{l, i, r}^{R}$, and the geometrical relationship in Fig. 1, the expression of the mean angle $\varphi_{l, i, r}$ and the corresponding angle spread $\Delta \varphi_{l, i}$ of the effective cluster in the $l$ th tap are given as

$$
\begin{aligned}
& \varphi_{l, i, r}=\left(\phi_{l, i-1, r}^{\prime R}+2 \phi_{l, i, r}^{\prime R}+\phi_{l, i+1, r}^{\prime R}\right) / 4= \pm\left[\arccos \left(2 \tau_{l-1}^{\prime} / \tau_{i, \max }^{\prime}-1\right)\right. \\
& \left.\quad+2 \arccos \left(2 \tau_{l}^{\prime} / \tau_{i, \max }^{\prime}-1\right)+\arccos \left(2 \tau_{l+1}^{\prime} / \tau_{i, \max }^{\prime}-1\right)\right] / 4 \\
& \Delta \varphi_{l, i}=\left|\left(\phi_{l, i-1, r}^{\prime R}-\phi_{l, i+1, r}^{\prime R}\right) / 4\right| \\
& \quad=\left[\arccos \left(2 \tau_{l-1}^{\prime} / \tau_{i, \max }^{\prime}-1\right)-\arccos \left(2 \tau_{l+1}^{\prime} / \tau_{i, \max }^{\prime}-1\right)\right] / 4
\end{aligned}
$$

Following the definition of the subintervals $G_{l}$ and some geometrical relationship shown in Fig. 1, we can determine the effective cluster in each tap according to the propagation delay $\tau_{l}^{\prime}$. The time-variant tap coefficient at the carrier frequency $f_{c}$ can be expressed as:

$$
\begin{aligned}
h_{l, o q}(t)=\lim _{M \rightarrow \infty} & \frac{1}{\sqrt{M}} \sum_{i=0}^{\Lambda_{l}-1} \sum_{r=1}^{R_{c}} \sum_{n=1}^{M} \exp \left\{j \left[\psi_{l, i, n}-2 \pi f_{c} \tau_{l, i, o q, n}\right.\right. \\
& \left.\left.+2 \pi f_{D} t \cos \left(\phi_{l, i, r, n}^{R}-\gamma\right)\right]\right\}
\end{aligned}
$$

with $\tau_{l, i, o q, n}=\xi_{l, i, q n}+\xi_{l, i, n o} / c$. Here, $\tau_{l, i . o q . n}$ is the travel time of the wave through the link $A_{q}-S_{l, i, n}-A_{o}$ scattered by the $n$th scatterer, $S_{l, i, n}, R_{c}$ is the number of effective cluster for one ring in each tap (here $R_{c}=2$ ), and $M$ is the number of effective scatterers, $S_{l, i, n}$, in the effective cluster, $S_{l, i}$. The AoA of the wave traveling from the $n$th scatterer in the effective cluster $S_{l, i}$ towards the MS is denoted by $\phi_{l, i, r, n}^{R}$. The phases $\psi_{l, i, n}$ are independent and identically distributed (i.i.d.) random variables with uniform distributions over $[0,2 \pi)$ and $f_{D}$ is the maximum Doppler frequency. As shown in [2], the distance $\xi_{l, i, q n}$ and $\xi_{l, i, n o}$ can be expressed as the function of $\phi_{l, i, r, n}^{R}$ as $\xi_{l, i, q n} \approx \zeta_{l, i, n}-\delta_{T}\left[\cos (\alpha)+\Theta_{l, i} \sin (\alpha) \sin \left(\phi_{l, i, r, n}^{R}\right)\right] / 2$ and $\xi_{l, i, n_{o}} \approx R_{l, i}-\delta_{R} \cos \left(\phi_{l, i, r, n}^{R}-\beta\right) / 2$, respectively, where $\zeta_{l, i, n} \approx D_{T R}+R_{l, i} \cos \left(\phi_{l, i, r, n}^{R}\right)$.

Since we assume that the number of effective scatterers in one effective cluster in this reference model tends to infinite (as shown in (5)), the discrete AoA, $\phi_{l, i, r, n}^{R}$ can be replaced by 


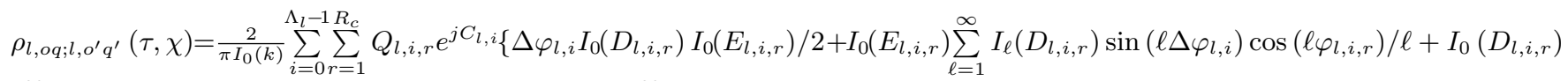

$$
\begin{aligned}
& \sum_{\ell^{\prime}=1}^{\infty}(-1)^{\ell^{\prime}} I_{\ell^{\prime}}\left(E_{l, i, r}\right) \sin \left(\ell^{\prime} \Delta \varphi_{l, i}\right) \cos \left(\ell^{\prime} \varphi_{l, i, r}+\frac{\ell^{\prime} \pi}{2}\right) / \ell^{\prime}+\sum_{\ell=1}^{\infty}(-1)^{\ell} I_{\ell}\left(D_{l, i, r}\right) I_{\ell}\left(E_{l, i, r}\right)\left[\Delta \varphi_{l, i} \cos \left(\frac{\ell \pi}{2}\right)+\sin \left(2 \ell \Delta \varphi_{l, i}\right) \cos \left(2 \ell \varphi_{l, i, r}+\frac{\ell \pi}{2}\right) /(2 \ell)\right] \\
& \left.+\sum_{\ell=1}^{\infty} \sum_{q=1}^{\infty}(-1)^{\ell^{\prime}} I_{\ell}\left(D_{l, i, r}\right) I_{\ell^{\prime}}\left(E_{l, i, r}\right)\left[\sin \left[\left(\ell+\ell^{\prime}\right) \Delta \varphi_{l, i}\right] \cos \left[\left(\ell+\ell^{\prime}\right) \varphi_{l, i, r}+\frac{\ell^{\prime} \pi}{2}\right] /\left(\ell+\ell^{\prime}\right)+\sin \left[\left(\ell-\ell^{\prime}\right) \Delta \varphi_{l, i}\right] \cos \left[\left(\ell-\ell^{\prime}\right) \varphi_{l, i, r}-\frac{\ell^{\prime} \pi}{2}\right] /\left(\ell-\ell^{\prime}\right)\right]\right\} \\
& (\ell \neq q)
\end{aligned}
$$

continuous random variables $\phi_{l, i, r}^{R}$. In the literature, many different scatterer distributions have been proposed to characterize the AoA $\phi_{l, i, r}^{R}$, such as the uniform and Gaussian probability density functions (PDFs). In this paper, the von Mises PDF) [3] is used, which can approximate all the above mentioned PDFs. The von Mises PDF is defined as $f(\phi) \triangleq \exp [k \cos (\phi-\mu)] / 2 \pi I_{0}(k)$, where $\phi \in[-\pi, \pi), I_{0}(\cdot)$ is the zeroth-order modified Bessel function of the first kind, $\mu \in[-\pi, \pi)$ accounts for the mean value of the angle $\phi$, and $k(k \geq 0)$ is a real-valued parameter that controls the angle spread of the angle $\phi$. To better characterize the AoA in one effective cluster $S_{l, i}$, we further modify the general expression of von Mises PDF as

$$
f_{c}\left(\phi_{l, i, r}^{R}\right)=Q_{l, i, r} \exp \left[k_{l, i, r} \cos \left(\phi_{l, i, r}^{R}-\varphi_{l, i, r}\right)\right] / 2 \pi I_{0}\left(k_{l, i, r}\right)
$$

where $\phi_{l, i, r}^{R} \in\left[\varphi_{l, i, r}-\Delta \varphi_{l, i}, \varphi_{l, i, r}+\Delta \varphi_{l, i}\right)$ and $Q_{l, i, r}$ is the normalization coefficient. Here we name the PDF in (6) the truncated von Mises PDF. Here, "truncated" means that the range of AoA in this PDF is only defined within a limited interval $\left[\varphi_{l, i, r}-\Delta \varphi_{l, i}, \varphi_{l, i, r}+\Delta \varphi_{l, i}\right)$. Therefore, the expression of the 'tapped' PDF of AoA in the lth tap of the proposed wideband multiple-ring channel model is given by

$$
\begin{aligned}
& f_{g}\left(\phi_{l, i, r}^{R}\right)=\sum_{i=0}^{\Lambda_{l}-1} \sum_{r=1}^{R_{c}} Q_{l, i, r} \exp \left[k_{l, i, r} \cos \left(\phi_{l, i, r}^{R}-\varphi_{l, i, r}\right)\right] 2 \pi I_{0}\left(k_{l, i, r}\right) \\
& \times w\left(\phi_{l, i, r}^{R}, \varphi_{l, i, r}-\Delta \varphi_{l, i}, \varphi_{l, i, r}+\Delta \varphi_{l, i}\right) \\
& \text { where } \quad \mathrm{w}(\phi, \mathrm{a}, \mathrm{b})= \begin{cases}1, & \text { if } a<\phi<b \\
0, & \text { otherwise. }\end{cases}
\end{aligned}
$$

Here, $Q_{l, i, r}$ are computed in such a way that the 'tapped' PDF $f_{d}\left(\phi_{l, i, r}^{R}\right)$ is equal to 1 , i.e., $\int_{0}^{2 \pi} f_{g}\left(\phi_{l, i, r}^{R}\right) d \phi_{l, i, r}^{R}=1$.

Note that the proposed wideband model allows us to consider the interaction of AoA, AoD, and ToA in a sensible manner. The interaction between the AoA and AoD is obtained in terms of the exact geometrical relationship, while the interaction between the AoA/AoD and ToA is calculated according to the TDL structure that allows us to investigate the correlation properties in each tap. Therefore, inspired by [7], the interaction between the AoA/AoD and ToA can be considered via setting the appropriate parameter $k_{l, i, r}$ for the PDF of AoA/AoD in each tap according to the PDF of ToA.

\section{NEW GENERIC STF CFS}

From the proposed model, in this section we derive the STF $\mathrm{CF}$ for each tap. The correlation properties of two arbitrary links $h_{o q}\left(t, \tau^{\prime}\right)$ and $h_{o^{\prime} q^{\prime}}^{\dagger}\left(t, \tau^{\prime}\right)$ at different frequency $f_{c}$ and $f_{c}^{\dagger}$ of a MIMO channel are completely determined by the correlation properties of $h_{o q}(t)$ and $h_{o^{\prime} q^{\prime}}^{\dagger}(t)$ in each tap since we assume that no correlations exist between the underlying processes in different taps. Therefore, we can restrict our investigations to the following $\operatorname{STF} \operatorname{CF} \rho_{l, o q ; l, o^{\prime} q^{\prime}}(\tau, \chi):=$
$\mathbf{E}\left[h_{l, o q}(t) h_{l, o^{\prime} q^{\prime}}^{\dagger *}(t+\tau)\right]$, where $(\cdot)^{*}$ denotes the complex conjugate operation and $\mathbf{E}[\cdot]$ designates the statistical expectation operator. Note that the above defined CF is a function of the time separation $\tau$, space separation $\delta_{T}$ and $\delta_{R}$, and frequency separation $\chi=f_{c}^{\dagger}-f_{c}$. As shown in the Appendix $\mathrm{A}$, the closed-form expression of 3-D STF CF $\rho_{l, o q ; l, o^{\prime} q^{\prime}}(\tau, \chi)$ can be presented as (8), shown at the top of the page, where $C_{l . i}=z \cos \alpha+\mathrm{X} T_{l . i}, D_{l, i, r}=a_{l, i, r}+j\left(\mathrm{X} U_{l, i}+y \cos \beta-x \cos \gamma\right)$, and $E_{l, i, r}=b_{l, i, r}+j\left(\mathrm{X} V_{l, i}+y \sin \beta+z \Theta_{l, i} \sin \alpha-x \sin \gamma\right)$ with $x=2 \pi f_{D} \tau, y=2 \pi f_{c} \delta_{R} / c, z=2 \pi f_{c} \delta_{T} / c, \mathrm{X}=2 \pi \chi / c$, $a_{l, i, r}=k_{l, i, r} \cos \varphi_{l, i, r}, \quad b_{l, i, r}=k_{l, i, r} \sin \varphi_{l, i, r}, T_{l, i}=$ $\left(\delta_{T} / 2\right) \cos \alpha+D_{T R}+R_{l, i}, \quad U_{l, i}=R_{l, i}+\left(\delta_{R} / 2\right) \cos \beta$, and $V_{l, i}=\left(\delta_{T} / 2\right) \Theta_{l, i} \sin \alpha+\left(\delta_{R} / 2\right) \sin \beta$. Consequently, the 3-D STF CF between $h_{o q}\left(t, \tau^{\prime}\right)$ and $h_{o^{\prime} q^{\prime}}^{\dagger}\left(t, \tau^{\prime}\right)$ can be shown as

$$
\rho_{o q, o^{\prime} q^{\prime}}(\tau, \chi)=\frac{1}{L} \sum_{l=0}^{\mathrm{L}-1} \rho_{l, o q ; l, o^{\prime} q^{\prime}}(\tau, \chi)
$$

It is clear that the derived STF CFs in (8) and (9) jointly consider the temporal, spatial, and frequency correlations. Note that (8) and (9) are the generic expressions which apply to the 3-D STF CF and the subsequently presented 2-D and 1-D CFs differ only in values of $C_{l, i}, D_{l, i}$, and $E_{l, i}$. The corresponding expressions of these three parameters for the degenerate 2-D and 1-D CFs can be easily obtained by setting relevant terms $\left(\tau, \delta_{T}\right.$ and $\delta_{T}$, and $\left.\chi\right)$ to zero.

Furthermore, a special case of the proposed model described by (1) is given when $L=1$ and $\Lambda_{l}=1$. Consequently, we have $h_{o q}\left(t, \tau^{\prime}\right)=h_{o q}(t) \delta\left(\tau^{\prime}\right)$, which is the impulse responses of the narrowband one-ring channel model. In such a case, the angle spread $\Delta \varphi_{l, i}=\pi$, which means the AoA range is over $[0,2 \pi)$. As shown in Appendix $\mathrm{B}$, we have

$$
\rho_{o q, o^{\prime} q^{\prime}}(\tau, \chi)=e^{j C} I_{0}\left[\left(D^{2}+E^{2}\right)^{1 / 2}\right] / I_{0}(k)
$$

which is essentially the same as the expression (6) given in [2] since $D^{2}+E^{2}=A-j B$ holds. Consequently, the derived STF CF also includes other CFs listed in [2] as special cases.

\section{Deterministic Simulation Model}

In this section, an efficient deterministic sum-of-sinusoid (SoS) simulation model is proposed also based on the TDL structure. The impulse response of the simulation model at the carrier frequency $f_{c}$ for the $A_{q}-A_{o}$ link are again composed of $L$ discrete taps according to

$$
\tilde{h}_{o q}\left(t, \tau^{\prime}\right)=\sum_{l=0}^{L-1} \tilde{h}_{l, o q}(t) \times \delta\left(\tau^{\prime}-\tau_{l}^{\prime}\right)
$$

In (11), the impulse responses $\tilde{h}_{l, o q}(t)$ is modeled by utilizing only a finite number of scatterers, $M$, and keeping all the model parameters fixed as

$$
\begin{gathered}
\tilde{h}_{l, o q}(t)=\frac{1}{\sqrt{M}} \sum_{i=0}^{\Lambda_{l}-1} \sum_{r=1}^{R_{c}} \sum_{n=1}^{M} \exp \left\{j \left[\tilde{\psi}_{l, i, n}-2 \pi f_{c} \tau_{l, i, o q, n}\right.\right. \\
\left.\left.+2 \pi f_{D} t \cos \left(\tilde{\phi}_{l, i, r, n}^{R}-\gamma\right)\right]\right\}
\end{gathered}
$$


where the phases $\tilde{\psi}_{l, i, n}$ are simply the outcomes of a random generator uniformly distributed over $[0,2 \pi)$, the discrete AoAs $\tilde{\phi}_{l, i, r, n}^{R}$ will be kept constant during simulation, and the other symbol definitions are the same as in (5). Therefore, we can analyze the properties of the deterministic channel simulator by time averages instead of statistical averages. The 3-D STF CF can be defined as $\tilde{\rho}_{l, o q ; l, o^{\prime} q^{\prime}}(\tau, \chi):=$ $\left\langle\tilde{h}_{l, o q}(t) \tilde{h}_{l, o^{\prime} q^{\prime}}^{\dagger *}(t+\tau)\right\rangle$ where $\langle\cdot\rangle$ denotes the time average operator. Substituting (12) into the aforementioned CF, we can get the closed-form STF CF as

$\tilde{\rho}_{l, o q ; l, o^{\prime} q^{\prime}}(\tau, \chi)=\frac{1}{M} \sum_{i=0}^{\Lambda_{l}-1} \sum_{r=1}^{R_{c}} \sum_{n=1}^{M} e^{j\left(C_{l, i}+P_{l, i} \cos \tilde{\phi}_{l, i, r, n}^{R}+J_{l, i} \sin \tilde{\phi}_{l, i, r, n}^{R}\right)}$

with $P_{l, i}=\mathrm{X} U_{l, i}+y \cos \beta-x \cos \gamma$ and $J_{l, i}=\mathrm{X} V_{l, i}+y \sin \beta+$ $z \Delta \sin \alpha-x \sin \gamma$ where $C_{l, i}, x, y, z, X, U_{l, i}$, and $V_{l, i}$ are the same as defined in (8). By analogy with (9), we can further get the 3-D STF CF between $\tilde{h}_{o q}\left(t, \tau^{\prime}\right)$ and $\tilde{h}_{o^{\prime} q^{\prime}}\left(t, \tau^{\prime}\right)$ as

$$
\tilde{\rho}_{o q, o^{\prime} q^{\prime}}(\tau, \chi)=\frac{1}{L} \sum_{l=0}^{L-1} \tilde{\rho}_{l, o q ; l, o^{\prime} q^{\prime}}(\tau, \chi) \text {. }
$$

Similar to (8) and (9), (13) and (14) are the generic expressions which apply to all the 3-D, 2-D, and 1-D CFs of the deterministic simulation model with different $C_{l, i}, P_{l, i}$, and $J_{l, i}$. Comparing the expressions of $D_{l, i}$ and $E_{l, i}$ with $P_{l, i}$ and $J_{l, i}$, respectively, we have $D_{l, i}=a_{l, i, r}+j P_{l, i}$ and $E_{l, i}=b_{l, i, r}+j J_{l, i}$. From (13) and (14), it is obvious that only $\left\{\tilde{\phi}_{l, i, r, n}^{R, n}\right\}_{n=1}^{M}$ needs to be determined for this deterministic simulation model.

The optimization method [11] is obtained here to calculate the model parameters $\left\{\tilde{\phi}_{l, i, r, n}^{R}\right\}_{n=1}^{M}$ of the deterministic simulation model based on corresponding properties of the reference model. The time $\mathrm{CF} \rho_{l, o q ; l, o q}(\tau)$, frequency $\mathrm{CF}$ $\rho_{l, o q ; l, o q}(\chi)$, and space $\mathrm{CF} \rho_{l, o q ; l, o^{\prime} q^{\prime}}$ are identified as key properties. Then the optimization method requires the numerical minimization of the following three $L_{p}$-norms: $E_{1}^{(p)}:=\left\{\int_{0}^{\tau_{\max }}\right.$ $\left.\left|\rho_{l, o q ; l, o q}(\tau)-\tilde{\rho}_{l, o q ; l, o q}(\tau)\right|^{p} d \tau / \tau_{\max }\right\}^{1 / p}, E_{2}^{(p)}:=\left\{\int_{0}^{\chi_{\max }}\right.$ $\left.\left|\rho_{l, o q ; l, o q}(\chi)-\tilde{\rho}_{l, o q ; l, o q}(\chi)\right|^{p} d \chi / \chi_{\max }\right\}^{1 / p}$, and $E_{3}^{(p)}:=\left\{\int_{0}^{\delta_{T}^{\max }}\right.$

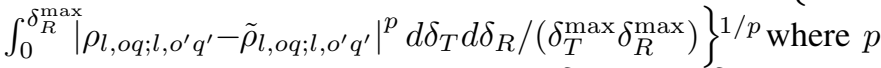
$=1,2, \ldots$ Note that $\tau_{\max }, \chi_{\max }$, and $\delta_{T}^{\max }$ and $\delta_{R}^{\max }$ define the upper limits of the ranges over which the approximations $\tilde{\rho}_{l, o q ; l, o q}(\tau) \approx \rho_{l, o q ; l, o q}(\tau), \tilde{\rho}_{l, o q ; l, o q}(\chi) \approx \rho_{l, o q ; l, o q}(\chi)$, and $\tilde{\rho}_{l, o q ; l, o^{\prime} q^{\prime}} \approx \rho_{l, o q ; l, o^{\prime} q^{\prime}}$ are of interest. For $\tilde{\rho}_{l, o q ; l, o q}(\chi)$ and $\tilde{\rho}_{l, o q ; l, o^{\prime} q^{\prime}}$, if we replace $\tilde{\phi}_{l, i, r, n}^{R}$ by $\tilde{\phi}_{l, i, r, n}^{\prime R}$ and $\tilde{\phi}_{l, i, r, n}^{\prime \prime R}$, respectively, the three error norms $E_{1}^{(p)}, E_{1}^{(p)}$, and $E_{3}^{(p)}$ can be minimized independently.

\section{NumERICAL RESUlts}

In this section, due to the page limit, we will only focus on the correlation properties of one tap $(l=2)$ with $\Lambda_{2}=4$ and $\left\{R_{2, i}\right\}_{i=0}^{3}=\{50,100,400,750\} m$ based on (8). The basic parameters are as follows: $f_{c}=5 \mathrm{GHz}, f_{D}=463 \mathrm{~Hz}$, $D_{T R}=2000 \mathrm{~m}, \alpha=\pi / 6, \beta=\pi / 3$, and $\gamma=7 \pi / 12$. The discrete COST $207 \mathrm{TU}$ channel model with $\left\{\tau_{l}^{\prime}\right\}_{l=0}^{5}=$ $\{0,0.2,0.5,1.6,2.3,5\} \mu s$ will be applied. For simplicity, we assume that $k_{l, i, r}=k$ for all effective clusters in the tap. Figs. 2 and 3 show the resulting frequency $\mathrm{CF}$ and space $\mathrm{CF}$, respectively. It is clear that the trend of relevant CFs is decreasing with the increase of the frequency separation $\chi$ and space separation $\delta_{R}$. As mentioned in many papers, Fig. 3 shows the AoA statistics (related to $k$ ) affect the spatial correlations. More interestingly, Fig. 2 illustrates the frequency correlations vary according to AoA statistics as well. It can also be observed that both spatial and frequency correlations increase with the increase of $k$ (i.e., with the decrease of angle spread of AoA). Moreover, we depict in both figures the CFs of the reference model with both the closed-form expression and the numerical integration method and the simulation model. Clearly, all these results match very well demonstrating the validity of our derivation and excellent performance of our simulation model. Fig. 3 also shows the impact of frequency separation on spatial correlations. It is clear that the frequency separation decreases the spatial correlation, while as expected (since the frequency correlation increase with the increase of $k$, as shown in Fig. 2) the reduction degree decreases with the increase of $k$. Therefore, we can conclude that in such a case, the resulting correlation is jointly contributed by the actual spatial correlation (i.e., due to the spatial distance/geometry of arrays only) and inherent frequency correlation. Note that this resulting correlation appears in both MIMO in a narrow sense and MIMO in a wide sense ${ }^{1}$. For MIMO in a narrow sense, the aforementioned correlation appears between two arbitrary subchannels of different antennas at different time instant, which are important for the appropriate design of STF coded MIMOOFDM systems [9]. While for MIMO in a wide sense, this correlation is extremely important for the sensible utilization of the space-frequency diversity [10].

\section{CONCLusions}

In this paper, we have extended the narrowband one-ring MIMO model to a new wideband multi-ring model for macrocell scenarios. According to the TDL structure of our model, the closed-formed expression of the 3-D STF CF for each tap has been derived. From the proposed wideband reference model, a deterministic SoS simulation model has been proposed. Numerical results have revealed the impact of AoA statistics on frequency correlations and the inherent frequency correlations within spatial correlations. Finally, the excellent agreement of correlations among the reference model based on our derived closed-form expressions, the reference model based on numerical integration methods, and the simulation model has validated our derivation of STF CF as well as the utility of the proposed simulation model.

\section{A. Derivations of (8)}

\section{APPENDIX}

Substituting (5) and (6) into the definition of the STF CF in this paper, and using our derived simplified expression of a well-known series $e^{z \sin \theta}=I_{0}(z)+2 \sum_{\ell=1}^{\infty}(-1)^{\ell} I_{\ell}(z) \cos [\ell \theta+\ell \pi / 2]$ and another well-known series [12, eq. 9.6.34, pp. 376], we have (15) shown at the top of next page, after the extensive

\footnotetext{
${ }^{1}$ MIMO in a narrow sense refers to a MIMO that exploits spatial multiplexing (or together with spatial diversity). In such a case, the same frequency is used at all antennas at the same time instant. While MIMO in a wide sense means any system with multiple antennas at both the transmitter and receiver.
} 


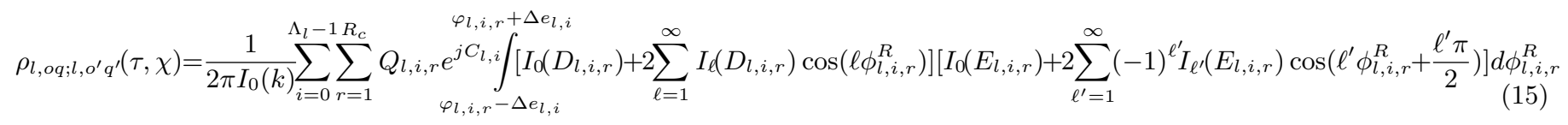

manipulations. Utilizing the basic knowledge of definite integral of trigonometric functions in [13], we can obtain the closed-form expression of (8).

\section{B. Derivations of (10)}

Substituting $\Delta \varphi_{l, i}=\pi$ and $\Lambda_{l}=R_{c}=1$ into (8), we have

$\rho_{l, o q ; l, o^{\prime} q^{\prime}}(\tau, \chi)=e^{j C}\left[I_{0}(D) I_{0}(E)+2 \sum_{\ell=1}^{\infty}(-1)^{\ell} I_{2 \ell}(D) I_{2 \ell}(E)\right] /{ }_{(16)}$

By making use of the Neumann's addition theorem [14, pp.359], the following equation can be obtained by further considering $J_{\ell}(j z)=j^{\ell} I_{\ell}(z)$, where $\ell$ is integral, and setting the angle $\phi=\pi / 2$ in the theorem

$$
I_{0}\left[\left(Z^{2}+z^{2}\right)\right]=I_{0}(Z) I_{0}(z)+2 \sum_{\ell=1}^{\infty}(-1)^{\ell} I_{2 \ell}(Z) I_{2 \ell}(z) .
$$

With the help of (16) and (17), (10) can be easily obtained.

\section{ACKNOWLEDGEMENTS}

We acknowledge the support from the Scottish Funding Council for the Joint Research Institute in Signal and Image Processing between the University of Edinburgh and HeriotWatt University which is a part of the Edinburgh Research Partnership in Engineering and Mathematics (ERPem).

\section{REFERENCES}

[1] A. Abdi, and M. Kaveh, "A space-time correlation model for multielement antenna systems in mobile fading channels," IEEE J. Select. Areas Commun., vol. 20, no. 3, pp. 550-560, Apr. 2002.

[2] X. Cheng, C.-X. Wang, and D. I. Laurenson,, "A generic space-timefrequency correlation model and its corresponding simulation model for MIMO wireless channels," 2nd EuCAP 2007, Edinburgh, Scotland, U.K., Nov. 2007, pp. 1-6.

[3] A. Abdi, J. A. Barger, and M. Kaveh, "A parametric model for the distribution of the angle of arrival and the associated correlation function and power spectrum at the mobile station," IEEE Trans. Veh. Technol.,vol. 51, no. 3, pp. 425-434, 2002.

[4] Z. Latinovic, A. Abdi, and Y. Bar-Ness, "A wideband space-time model for MIMO mobile fading channels," IEEE WCNC 2003, New Orleans, LA, USA, Mar. 2003, pp. 338-342.

[5] M. Pätzold and B. O. Hogstad, "A Wideband Space-Time MIMO Channel Simulator Based on the Geometrical One-Ring Model," IEEE VTC 2006Fall, Montreal, Canada, Sept. 2006, pp. 1-6.

[6] C.-X. Wang, M. Pätzold, and Q. Yao, "Stochastic modelling and simulation of frequency correlated wideband fading channels," IEEE Trans. Veh. Technol., vol. 56, no. 3, pp. 1050-1063, May 2007.

[7] C. C. Chong, C. M. Tan, D. I. Laurenson, S. McLaughlin, M. A. Beach, and A. R. Nix, "A new statistical wideband spatio-temporal channel model for 5-GHz band WLAN systems," IEEE J. Select. Areas Commun., vol. 21, no. 2, pp. 139-150, Feb. 2003.

[8] J. Foersler, "Channel modeling sub-committee reprot (final)," IEEE P802.15-02/490r1-SG3a, Feb. 2003.

[9] H. Bölcskei, M. Borgmann, and A. Paulraj, "Impact of the propagation environment on the performance of space-frequency code MIMO-OFDM," IEEE Journal on Selected Areas in Commun., vol. 21, no. 3, pp. 427439, 2003.

[10] M. Kalkan and R. H. Clarke, "Prediction of the space-frequency correlation function for base station diversity reception," IEEE Trans. Veh. Technol., vol. 46, no. 1, pp. 176-184, Feb. 1997.

[11] M. Pätzold, Mobile Fading Channels. Chichester: John Wiley \& Sons, 2002.
[12] M. Abramowitz and I. A. Stegun, Handbook of Mathematical Functions with Formulas, Graphs and Mathematical Tables. New York: Dover, 1965.

[13] I. S. Gradshteyn and I. M. Ryzhik, Table of Integrals, Series, and Products. 5th ed, A. Jeffrey, Ed. San Diego, CA: Academic, 1994.

[14] G. N. Watson, A Treatise of the Theory of Bessel Functions. 2nd ed. Cambridge, 1952.

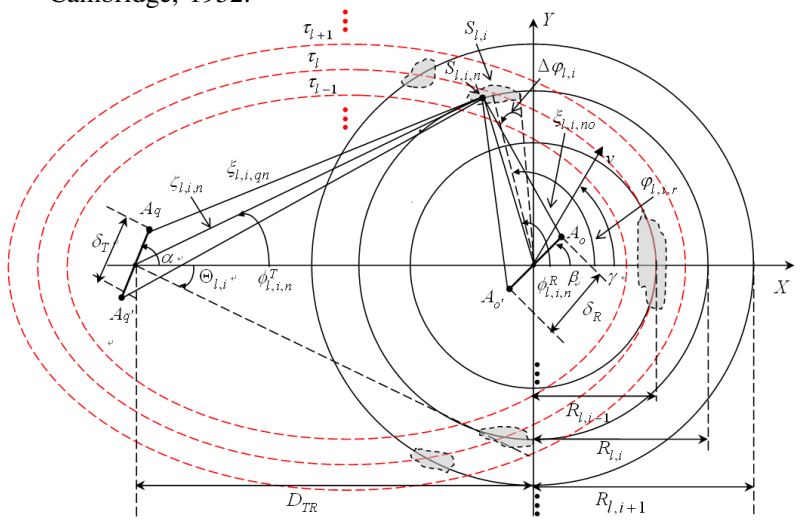

Fig. 1. A new wideband multiple-ring MIMO channel model.

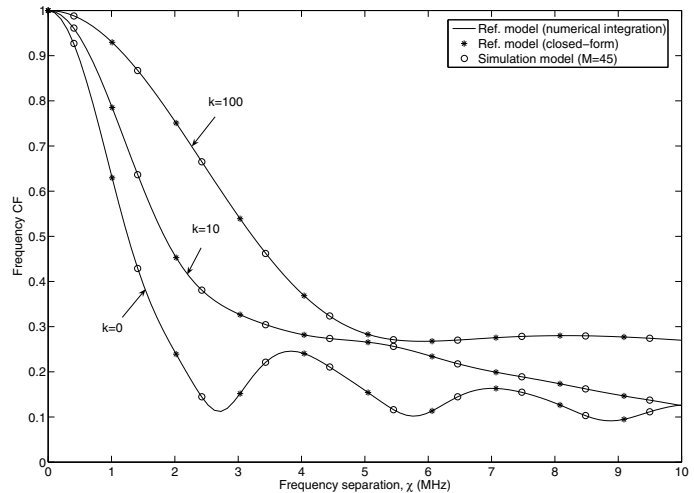

Fig. 2. The frequency CFs $\left|\rho_{l, o q ; l, o q}(\chi)\right|$ (reference model) and $\left|\tilde{\rho}_{l, o q ; l, o q}(\chi)\right|$ (simulation model, $M=45$ ) for different values of the parameter $k$.
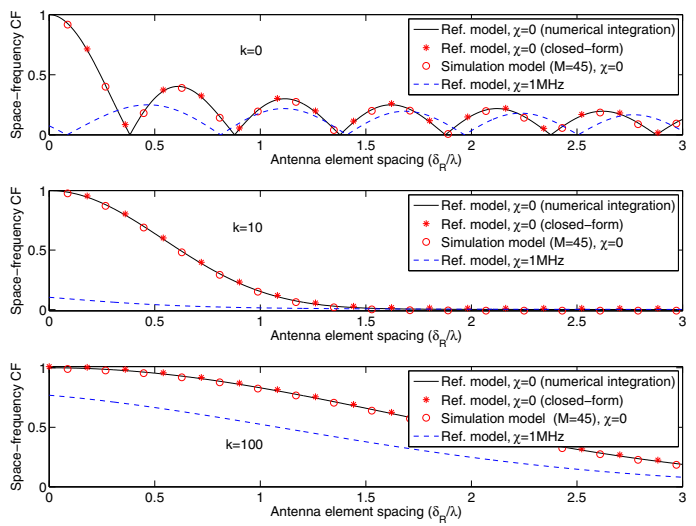

Fig. 3. The space-frequency CFs $\left|\rho_{l, o q ; l, o^{\prime} q^{\prime}}\right|$ (reference model) and $\left|\tilde{\rho}_{l, o q ; l, o^{\prime} q^{\prime}}\right|$ (simulation model, $M=45$ ) for different values of the parameter $k$. 